\title{
Nonradiative proton-deuteron fusion in stellar plasma
}

\author{
S. A. Rakityansky*, S. A. Sofianos, L. L. Howell, M. Braun \\ Physics Department, University of South Africa, P.O.Box 392, Pretoria 0001, South Africa \\ V. B. Belyaev \\ Joint Institute for Nuclear Research,Dubna, 141980, Russia
}

(November 12, 2018)

\begin{abstract}
The nuclear reaction $e+p+d \rightarrow{ }^{3} \mathrm{He}+e$ is considered at thermonuclear energies. The motion of the electron is treated within the adiabatic approximation and the $p d$ scattering state is constructed in the form of an antisymmetrized product of the bound state wave function of the deuteron and of the wave function of the $p d$ relative motion. The latter is calculated using an effective $p d$ potential constructed via the Marchenko inverse scattering method. The bound state wave function of ${ }^{3} \mathrm{He}$ is obtained using Faddeev-type integrodifferential equations. The reaction rate thus obtained for the solar interior conditions is approximately $10^{-4}$ of the corresponding rate for the radiative capture $p d \rightarrow{ }^{3} \mathrm{He} \gamma$.
\end{abstract}

PACS number: 21.45.+v, 95.30.-k, 97.10.Cv

Key words: Thermonuclear reactions, fusion, stellar plasma.

*Permanent address: Joint Institute for Nuclear Research,Dubna, 141980, Russia 


\section{INTRODUCTION}

Burning of hydrogen in the main sequence stars mainly occurs through the $p p$-chain which begins with the reaction $p p \longrightarrow e^{+} d \nu$. It is generally accepted [1] that the second step of this chain is the radiative capture of protons by deuterons,

$$
p+d \longrightarrow{ }^{3} \mathrm{He}+\gamma \text {. }
$$

However, due to the high densities in stars, the helium nuclei and other intermediate products of the $p p$-chain can emerge not only from two-body but from three-body initial states as

well. Thus, for example, ${ }^{3} \mathrm{He}$ can be formed in the radiative capture (1) as well as in the three-body nonradiative fusion process

$$
e+p+d \longrightarrow{ }^{3} \mathrm{He}+e .
$$

The knowledge of the production rates of various nuclei in the stellar plasma is very important, not only in understanding the production of energy in stars but also in explaining the abundance of the elements observed in nature, and in describing events during the first thousand seconds of the evolution of the universe which were predominantly determined by the nucleosynthesis of light elements [2]. The abundance of light elements in the Universe, together with the Hubble expansion and the relic backround radiation are experimental evidences for the idea of the hot origin of the Universe in the Big Bang process which in turn is closely related to the Grand Unification and QCD theories [2, 3].

Values of the primordial abundance of light elements put some constraints on the baryon density of the Universe as well as on the number of species of light particles. For instance, present theoretical estimates for the primordial abundance of $d,{ }^{3} \mathrm{He},{ }^{4} \mathrm{He}$, and ${ }^{7} \mathrm{Li}$ can be in agreement with the corresponding experimental values only if the number of the neutrino species is $N_{\nu} \leq 3.9$ [4]. Furthermore, from the theory of nucleosynthesis one can derive a stringent limit to the existence of new light particles and even a bound to the mass of the $\tau$-neutrino, namely, between $0.5 \mathrm{MeV}$ and $30 \mathrm{MeV}$ [5.6].

Any theory on the evolution of the universe or nucleosynthesis must deal with the total rates of nuclei production. In this respect the thermonuclear reactions with two-body initial states, such as the reaction (四) have been extensively investigated [7]. However, the role of the three-body mechanism in nucleosynthesis has not yet been properly studied despite the fact that the three-body processes have different selection rules and due to this it can have an influence on the production of light nuclei.

The aim of this work is to estimate the relative significance of the nonradiative process (2) in comparison with the radiative capture (1) in the stellar $p p$-chain. In stellar plasma nuclei are surrounded by electron gas which has a twofold influence on nuclear fusion processes. We can distinguish between static and dynamic electronic effects [8]. The former is the screening of the Coulomb repulsion between the nuclei, and the latter stems from the coupling between the electronic and nucleonic degrees of freedom. Due to this coupling, energy and angular momentum can be transferred from the nucleons to the electrons according 
to the prevailing conservation laws. These dynamic electronic effects can pave the way for a variety of possible fusion reactions in the plasma, which otherwise are forbidden.

The nonradiative fusion (2) is an example where these effects are manifested. The electron which is in the vicinity of the $p d$-pair while they are interacting can carry away the excess energy, leaving the three nucleons in a bound state. This is a kind of Auger transition in the continuous spectrum. Since electrons move much faster than nucleons, in considering the scattering of an electron by a $p d$-pair, its motion can be treated within the adiabatic approximation. In this approximation the nucleons are considered as being fixed at their spatial positions during the electron scattering and thus the corresponding amplitude will depend on the nucleon coordinates parametrically. The physical amplitude can then be obtained by averaging over these coordinates with the help of the wave functions describing the initial and final configurations of the nucleons. To obtain the amplitude of the reaction (2) we shall average the fixed-scatterer amplitude over the $p d$ scattering state and the ${ }^{3} \mathrm{He}$ bound state.

The paper is organized as follows. In Sec. II we describe our formalism and outline the procedure employed to evaluate the various ingredients used to obtain the reaction rate. In Sec. III we present our results and conclusions. Some details concerning the derivation of the reaction rate formula are given in the Appendix.

\section{FORMALISM}

We are concerned with electron collisions with nuclei in a stellar plasma consisting of protons, deuterons, and electrons. Let $\Psi_{\mathbf{k}}$ be the wave function of the relative motion of the $p d$-pair with momentum $\mathbf{k}$ and let $\mathbf{p}$ be the momentum of the electron with respect to this pair. The reaction rate for the collision process (2) per unit volume per second is defined by [9]

$$
\mathcal{R}\left(\mathbf{k}, \mathbf{p} \longrightarrow \mathbf{p}^{\prime}\right)=\delta\left(E_{f}-E_{i}\right)(2 \pi)^{7}\left|\left\langle\Psi_{3}, \mathbf{p}^{\prime}|T| \Psi_{\mathbf{k}}, \mathbf{p}\right\rangle\right|^{2} n_{p} n_{d} n_{e}
$$

where the states in the continuum are normalized as

$$
\left\langle\Psi_{\mathbf{k}^{\prime}}, \mathbf{p}^{\prime} \mid \Psi_{\mathbf{k}}, \mathbf{p}\right\rangle=\delta\left(\mathbf{k}^{\prime}-\mathbf{k}\right) \delta\left(\mathbf{p}^{\prime}-\mathbf{p}\right) .
$$

$T$ is the transition operator, $\Psi_{3}$ is the bound state wave function of ${ }^{3} \mathrm{He}$ and $n_{p}, n_{d}$, and $n_{e}$ represent particle densities.

In stellar plasmas the momenta $\mathbf{k}$ and $\mathbf{p}$ are distributed according to Maxwell's law

$$
\begin{aligned}
& N_{\mathbf{k}}=(2 \pi \mu \kappa \Theta)^{-3 / 2} \exp \left(-\frac{k^{2}}{2 \mu \kappa \Theta}\right), \\
& N_{\mathbf{p}}=(2 \pi m \kappa \Theta)^{-3 / 2} \exp \left(-\frac{p^{2}}{2 m \kappa \Theta}\right),
\end{aligned}
$$

where $N_{\mathbf{k}}$ and $N_{\mathbf{p}}$ are the probability densities, $\mu$ is the proton-deuteron reduced mass, $m$ is the electron mass, $\kappa$ is the Boltzmann constant, and $\Theta$ is the plasma temperature. Thus 
the reaction rate (3) must be averaged over the initial momenta $\mathbf{k}$ and $\mathbf{p}$ and integrated over the final momentum $\mathbf{p}^{\prime}$, i.e.,

$$
\langle\mathcal{R}\rangle=\iiint d \mathbf{k} d \mathbf{p} d \mathbf{p}^{\prime} \mathcal{R}\left(\mathbf{k}, \mathbf{p} \longrightarrow \mathbf{p}^{\prime}\right) N_{\mathbf{k}} N_{\mathbf{p}}
$$

In what follows we shall discuss the various ingredients of this formula in somewhat more detail.

\section{A. Transition operator}

Consider a four-body system consisting of an electron and three nucleons described by the Jacobi vectors shown in Fig. I. The total Hamiltonian of this system consists of three terms, namely,

$$
H=H_{3}+h_{0}+V_{e}
$$

where $H_{3}$ is the total nuclear Hamiltonian, $h_{0}$ is the kinetic energy operator for the free motion of the electron with respect to the center of mass of the nucleons, and $V_{e}$ is the sum of the Coulomb potentials between the electron and nucleons

$$
V_{e}=-\frac{\hat{Z}_{1} e^{2}}{r_{1}}-\frac{\hat{Z}_{2} e^{2}}{r_{2}}-\frac{\hat{Z}_{3} e^{2}}{r_{3}} .
$$

Here $r_{i}$ are the distances between the electron and the nucleons, and $\hat{Z}_{i}$ is the charge-operator for the $i$-th nucleon with

$$
\hat{Z}_{i} \mid 1 / 2, \tau_{i}>=\left\{\begin{array}{lll}
1, & \text { if } & \tau_{i}=+1 / 2 \\
0, & \text { if } & \tau_{i}=-1 / 2
\end{array}\right.
$$

where $\mid 1 / 2, \tau_{i}>$ is the isotopic state of the $i$-th nucleon with $\tau_{i}$ being the third component of its isospin.

Since the motion of nucleons is much slower than that of the electron, the $T$-matrix describing the electron scattering can be found with the help of the fixed-scatterer approximation

$$
T(z)=V_{e}+V_{e} G_{0}(z) T(z),
$$

where $z$ is the total energy and

$$
G_{0}(z)=\left(z-h_{0}\right)^{-1}
$$

is the free Greens' function. Parametrical dependence of the potential (6) on the nuclear Jacobi vectors $\{\boldsymbol{\rho}, \mathbf{r}\}$ and on the nuclear isospin state $\mid \eta>$ of the three nucleons, makes the fixed-scatterer $T$-matrix (8) also parametrically dependent on them,

$$
\left\langle\boldsymbol{\rho}^{\prime}, \mathbf{r}^{\prime} ; \eta^{\prime} ; \mathbf{p}^{\prime}|T(z)| \boldsymbol{\rho}, \mathbf{r} ; \eta ; \mathbf{p}\right\rangle=\delta_{\eta \eta^{\prime}} \delta\left(\boldsymbol{\rho}^{\prime}-\boldsymbol{\rho}\right) \delta\left(\mathbf{r}^{\prime}-\mathbf{r}\right) T_{\mathbf{p}^{\prime} \mathbf{p}}^{\eta}(\boldsymbol{\rho}, \mathbf{r} ; z),
$$


where $\mathbf{p}$ and $\mathbf{p}^{\prime}$ are the initial and final momenta of the electron. The physical $T$-matrix is obtained by the averaging,

$$
\left\langle\Psi_{3}, \mathbf{p}^{\prime}|T(z)| \Psi_{\mathbf{k}}, \mathbf{p}\right\rangle=\sum_{\chi \eta} \iint d \boldsymbol{\rho} d \mathbf{r} \psi_{3}^{\chi \eta *}(\boldsymbol{\rho}, \mathbf{r}) T_{\mathbf{p}^{\prime} \mathbf{p}}^{\eta}(\boldsymbol{\rho}, \mathbf{r} ; z) \psi_{\mathbf{k}}^{\chi \eta}(\boldsymbol{\rho}, \mathbf{r})
$$

where $\psi_{\mathbf{k}}^{\chi \eta}$ and $\psi_{3}^{\chi \eta}$ are the spatial parts of the three-nucleon wave functions corresponding to the spin-isospin state $|\chi \eta\rangle$,

$$
\left.|\chi \eta>\equiv|\left(\left(s_{1} s_{2}\right) s_{12}, s_{3}\right) \frac{1}{2} \sigma ;\left(\left(t_{1} t_{2}\right) t_{12}, t_{3}\right) \frac{1}{2} \tau\right\rangle
$$

i.e.,

$$
\psi_{\mathbf{k}}^{\chi \eta}(\boldsymbol{\rho}, \mathbf{r})=\left\langle\boldsymbol{\rho}, \mathbf{r} ; \chi \eta \mid \Psi_{\mathbf{k}}\right\rangle
$$

and

$$
\psi_{3}^{\chi \eta}(\boldsymbol{\rho}, \mathbf{r})=\left\langle\boldsymbol{\rho}, \mathbf{r} ; \chi \eta \mid \Psi_{3}\right\rangle
$$

\section{B. Proton-deuteron scattering state}

The thermonuclear energies we are considering are far below the threshold of the deuteron break-up and we can therefore neglect the three-nucleon continuous spectrum $(1+1+1)$ and construct the $(1+2)$ scattering state in the form of the antisymmetrized product of the proton-deuteron relative motion wave function $\varphi_{\mathbf{k}}$ and the deuteron wave function $\psi_{2}$,

$$
\Psi_{\mathbf{k}}=\mathcal{A}\left\{\varphi_{\mathbf{k}} \psi_{2} \chi \eta\right\}
$$

Since the deuteron wave function is antisymmetric, the antisymmetrizer $\mathcal{A}$ involves only the permutations $P_{23}$ and $P_{13}$,

$$
\mathcal{A}=\frac{1}{\sqrt{3}}\left(1-P_{23}-P_{13}\right)
$$

Both $\varphi_{\mathbf{k}}$ and $\psi_{2}$ are assumed to have $S$-wave components only with the total spin of the $n d$-system equal to $1 / 2$. Before the antisymmetrization, the spin-isospin states of the $n d-$ system are $|\chi \eta\rangle=\left|\left(\left(s_{1} s_{2}\right) 1 s_{3}\right) \frac{1}{2} \sigma ;\left(\left(t_{1} t_{2}\right) 0 t_{3}\right) \frac{1}{2} \tau\right\rangle$. Thus after the nucleon permutations we obtain

$$
\begin{aligned}
\left\langle\boldsymbol{\rho}, \mathbf{r} \mid \Psi_{\mathbf{k}}\right\rangle & =\frac{1}{\sqrt{3}}\left\{\varphi_{\mathbf{k}}(\boldsymbol{\rho}) \psi_{2}(\mathbf{r})\left|\left(\left(s_{1} s_{2}\right) 1 s_{3}\right) \frac{1}{2} \sigma ;\left(\left(t_{1} t_{2}\right) 0 t_{3}\right) \frac{1}{2} \tau\right\rangle\right. \\
& -\varphi_{\mathbf{k}}\left(-\frac{1}{2} \boldsymbol{\rho}+\frac{3}{4} \mathbf{r}\right) \psi_{2}\left(\boldsymbol{\rho}+\frac{1}{2} \mathbf{r}\right)\left|\left(\left(s_{1} s_{3}\right) 1 s_{2}\right) \frac{1}{2} \sigma ;\left(\left(t_{1} t_{3}\right) 0 t_{2}\right) \frac{1}{2} \tau\right\rangle \\
& \left.-\varphi_{\mathbf{k}}\left(-\frac{1}{2} \boldsymbol{\rho}-\frac{3}{4} \mathbf{r}\right) \psi_{2}\left(\frac{1}{2} \mathbf{r}-\boldsymbol{\rho}\right)\left|\left(\left(s_{3} s_{2}\right) 1 s_{1}\right) \frac{1}{2} \sigma ;\left(\left(t_{3} t_{2}\right) 0 t_{1}\right) \frac{1}{2} \tau\right\rangle\right\} .
\end{aligned}
$$

The spatial components $\psi_{\mathbf{k}}^{\chi \eta}(\boldsymbol{\rho}, \mathbf{r})$, Eq. (11]), can be obtained by projecting on to the spinisospin states (10). 
To find the deuteron wave function $\psi_{2}(\mathbf{r})$ we solve the two-body Schrödinger equation with the Malfliet-Tjon I-III [10 $N N$-potential. In order to obtain the relative motion wave function $\varphi_{\mathbf{k}}(\boldsymbol{\rho})$ the two-body scattering problem is solved using the Jost function method proposed in Ref. [11] with an effective proton-deuteron potential $V_{p d}(\rho)$ which consists of two terms:

$$
V_{p d}(\rho)=V_{c}(\rho) \exp \left(-\frac{\rho}{\rho_{D}}\right)+V_{s}(\rho)
$$

The first term describes the proton-deuteron Coulomb interaction. Since deuteron is not a point-like particle, we take into account the spherically symmetric distribution of its charge in space by using

$$
V_{c}(\rho)=\frac{4 \pi e^{2}}{\rho} \int_{0}^{2 \rho} d r\left[r \psi_{2}(r)\right]^{2} .
$$

At large $\rho$ (beyond the deuteron radius), $V_{c}(\rho)$ coincides with the simple Coulomb potential $e^{2} / \rho$. However at small $\rho$ it behaves quite differently. In particular, $V_{c}(\rho)$ is not singular at $\rho=0$, but instead $V_{c}(0)=0$. The exponential factor stems from electron screening and follows from the standard Debye-Hückel theory [12]. For the Debye radius we use the value

$$
\rho_{D}=21800 \mathrm{fm}
$$

which corresponds to the solar plasma conditions and is typical for other stars [\&].

The $V_{s}(\rho)$ is the strong (nuclear) $p d$ interaction and we constructed it using the $\ell$ - dependent Marchenko inverse scattering method [13,14] which we briefly describe next.

\section{Marchenko Inverse Scattering method}

In the Marchenko inverse scattering method a unique, energy-independent, $\ell$-dependent, local potential $V_{\ell}(\rho)$ can be constructed which is phase equivalent to the $n d$ doublet channel effective interaction at all energies. This potential is obtained from

$$
V_{\ell}(\rho)=-2 \frac{\mathrm{d}}{d \rho} K_{\ell}(\rho, \rho)
$$

where the kernel $K_{\ell}\left(\rho, \rho^{\prime}\right)$ obeys the Marchenko fundamental equation

$$
K_{\ell}\left(\rho, \rho^{\prime}\right)+F_{\ell}\left(\rho, \rho^{\prime}\right)+\int_{r}^{\infty} K_{\ell}\left(\rho, \rho^{\prime \prime}\right) F_{\ell}\left(\rho^{\prime \prime}, \rho^{\prime}\right) d \rho^{\prime \prime}=0
$$

Here, $\rho$ is the relative distance between the neutron and deuteron and is canonically conjugate to the relative momentum $k$. The driving term $F_{\ell}\left(\rho, \rho^{\prime}\right)$ is given by

$$
F_{\ell}\left(\rho, \rho^{\prime}\right)=\frac{1}{2 \pi} \int_{-\infty}^{+\infty} w_{\ell}^{+}(k \rho)\left[1-S_{\ell}(k)\right] w_{\ell}^{+}\left(k \rho^{\prime}\right) \mathrm{d} k+A_{\ell} w_{\ell}^{+}\left(b_{\ell} \rho\right) w_{\ell}^{+}\left(b_{\ell} \rho^{\prime}\right) .
$$

$S_{\ell}(k)$ is the $\mathrm{S}$-matrix for the specific partial wave $\ell$, and the function $w_{\ell}^{+}(z)$ is related to the spherical Hankel function $h_{\ell}^{(+)}(z)$ by 


$$
w_{\ell}^{+}(z)=i e^{i \pi \ell} z h_{\ell}^{(+)}(z) .
$$

Furthermore, $A_{\ell}$ is the so-called asymptotic bound state normalisation constant, while $b_{\ell}=\sqrt{-4 M E_{b}^{\ell} / 3}, E_{b}^{\ell}$ being the three-body bound state energy and $M$ the nucleon mass.

As is apparent from its definition (18) the evaluation of $F_{\ell}\left(\rho, \rho^{\prime}\right)$ requires the knowledge of the $\mathrm{S}$-matrix for all real energies from the elastic scattering threshold to infinity, together with the reflection property $S_{\ell}(-k)=1 / S_{\ell}(k)$, as well as the binding energy and the corresponding asymptotic bound state normalisation constant. It is greatly simplified by choosing a rational (Bargmann-type) parametrisation

$$
S_{\ell}(k)=\frac{k+i b_{\ell}}{k-i b_{\ell}} \prod_{n=1}^{N_{\ell}} \frac{k+\alpha_{n}^{\ell}}{k-\alpha_{n}^{\ell}}
$$

The number $N_{\ell}$ is to be taken odd to satisfy the requirement $S_{\ell} \rightarrow 1$ for $k \rightarrow 0$. The $\alpha_{n}^{\ell}$ are complex numbers used to fit the (numerically) given $\mathrm{S}$-matrix.

With the choice (20) the integration in Eq. (18) can be easily performed analytically, the result being

$$
F_{\ell}\left(\rho, \rho^{\prime}\right)=-i \sum_{m=1}^{N_{\ell}^{u}} R_{m}^{\ell} w_{\ell}^{+}\left(\alpha_{m}^{\ell} \rho\right) w_{\ell}^{+}\left(\alpha_{m}^{\ell} \rho^{\prime}\right)+A_{\ell} w_{\ell}^{+}\left(b_{\ell} \rho\right) w_{\ell}^{+}\left(b_{\ell} \rho^{\prime}\right) .
$$

Here, $R_{m}^{\ell}$ are the coefficients of the residues of the integrand for the $N_{\ell}^{u}$ poles of (20) which are lying in the upper half complex $\mathrm{k}$-plane (excluding the one corresponding to the bound state at $\left.k=i b_{\ell}\right)$. The separable form of $F_{\ell}\left(\rho, \rho^{\prime}\right)$ provides us with an algebraic solution of the integral equation (17) for the kernel $K_{\ell}\left(\rho, \rho^{\prime}\right)$ from which the potential can be obtained via Eq. (16).

In order that the potential be unique, the asymptotic bound state normalisation constant $A_{\ell}$ must be given a definite value. We choose it to be [14

$$
A_{\ell}=i \frac{f_{\ell}\left(-i b_{\ell}\right)}{f_{\ell}^{\prime}\left(i b_{\ell}\right)}
$$

where $f_{\ell}$ is the Jost function, and $f_{\ell}^{\prime}(k)=d f_{\ell}(k) / d k$.

\section{Wave function of ${ }^{3} \mathrm{He}$}

The integrodifferential equation approach to few- and many body systems developed by Fabre de la Ripelle and collaborators [15, 17, 18] is used to construct the bound state wave function of ${ }^{3} \mathrm{He}$. In this method the potential $U$ is written as a sum of two-body interactions

$$
U=\sum_{i<j} V\left(r_{i j}\right)
$$

and the three-body bound state wave function is written as a sum of two-body amplitudes 


$$
\psi_{3}=H_{\left[L_{m}\right]}(\mathbf{x}) \sum_{i<j} F\left(r_{i j}, r_{0}\right)
$$

where $H_{\left[L_{m}\right]}(\mathbf{x})$ is the harmonic polynomial of lowest degree $\left[L_{m}\right]$ occuring in the harmonic polynomial expansion of the wave function, and $\mathbf{x}$ represents the nucleon coordinates $\mathbf{x}_{i}$ with $r_{i j}=\left|\mathbf{x}_{i}-\mathbf{x}_{j}\right|$, and $r_{0}=\left[2 / 3 \sum_{i<j} \mathbf{r}_{i j}^{2}\right]^{1 / 2}$ is the hyperradius.

With the above expansions one has to solve, instead of the Schrödinger equation, the Faddeev-type equation for the amplitude $F\left(r_{i j}, r_{0}\right)$

$$
(T-E) H_{\left[L_{m}\right]}(\mathbf{x}) F\left(r_{i j}, r_{0}\right)=-V\left(r_{i j}\right) H_{\left[L_{m}\right]}(\mathbf{x}) \sum_{k<\ell} F\left(r_{k \ell}, r_{0}\right) .
$$

A solution of this equation will be an approximate solution of the Schrödinger equation for two-body amplitudes where pairs are in $S$-states. Another Faddeev-type equation can be obtained by extracting the hypercentral potential $V_{\left[L_{m}\right]}\left(r_{0}\right)$ of $V\left(r_{i j}\right)$ [15] and by writing

$$
\begin{aligned}
\left(T+\frac{A(A-1)}{2} V_{\left[L_{m}\right]}\left(r_{0}\right)\right. & -E) H_{\left[L_{m}\right]}(\mathbf{x}) F\left(r_{i j}, r_{0}\right) \\
& =-\left[V\left(r_{i j}\right)-V_{\left[L_{m}\right]}\left(r_{0}\right)\right] H_{\left[L_{m}\right]}(\mathbf{x}) \sum_{k<\ell} F\left(r_{k \ell}, r_{0}\right) .
\end{aligned}
$$

This equation forms the basis of the integrodifferential equation approach (IDEA) to fewand many-body systems [16] and takes into account in an approximate way, via the hypercentral potential $V_{\left[L_{m}\right]}\left(r_{0}\right)$, the effects of the coupling between the orbitals $\ell \neq 0$ of the spectator particle and the interacting pair. For $\left[L_{m}\right]=0$ then $H_{\left[L_{m}\right]}(\mathbf{x})=1$. We notice that by summing over all pairs one generates the Schrödinger equation but here the two-body potential is the residual interaction on the right hand side of (26).

We assume that we have a central spin-dependent nucleon-nucleon potential of the form

$$
V^{+}\left(r_{i j}, \sigma, \tau\right)=V^{1+}\left(r_{i j}\right) P_{i j}^{1+}+V^{3+}\left(r_{i j}\right) P_{i j}^{3+}
$$

where the projection operators $P_{i j}^{1+}$ and $P_{i j}^{3+}$ are acting on the singlet- and triplet-even states respectively. In order to proceed two further steps are required. In the first step $F\left(r_{i j}, r_{0}\right)$ is written,

$$
F\left(r_{i j}, r_{0}\right)=P\left(\zeta_{i j}, r_{0}\right) / r_{0}^{(D-1) / 2}
$$

where $\zeta_{i j}=2 r_{i j}^{2} / r_{0}^{2}-1$ and $D=3 A-3$. In the second step Eq. (26) is projected on the $r_{i j}$ space to give two coupled integrodifferential equations

$$
\begin{aligned}
{\left[\frac{\hbar^{2}}{m} \nabla_{0}^{2}-\frac{A(A-1)}{2} V_{0}\left(r_{0}\right)+E\right] P_{0}^{S}\left(\zeta, r_{0}\right) } & =\left[\frac{V^{1+}+V^{3+}}{2}-V_{0}\left(r_{0}\right)\right] \Pi_{0}^{S}\left(\zeta, r_{0}\right) \\
& +\left[\frac{V^{1+}-V^{3+}}{2}\right] \Pi_{0}^{S^{\prime}}\left(\zeta, r_{0}\right) \\
{\left[\frac{\hbar^{2}}{m} \nabla_{0}^{2}-\frac{A(A-1)}{2} V_{0}\left(r_{0}\right)+E\right] P_{0}^{S^{\prime}}\left(\zeta, r_{0}\right) } & =\left[\frac{V^{1+}+V^{3+}}{2}-V_{0}\left(r_{0}\right)\right] \Pi_{0}^{S^{\prime}}\left(\zeta, r_{0}\right) \\
& +\left[\frac{V^{1+}-V^{3+}}{2}\right] \Pi_{0}^{S}\left(\zeta, r_{0}\right),
\end{aligned}
$$


where

$$
\Pi_{0}^{n}\left(\zeta, r_{0}\right)=P_{0}^{n}\left(\zeta, r_{0}\right)+\int_{-1}^{+1} f_{(0)}^{n}\left(\zeta, \zeta^{\prime}\right) P_{0}^{n}\left(\zeta^{\prime}, r_{0}\right) d \zeta^{\prime}
$$

with $n=S, S^{\prime}$.

The $\nabla_{0}^{2}$ is given by

$$
\nabla_{0}^{2}=\frac{\partial^{2}}{\partial r_{0}^{2}}-\frac{\mathcal{L}_{0}\left(\mathcal{L}_{0}+1\right)}{r_{0}^{2}}+\frac{4}{r_{0}^{2}} \frac{1}{W_{0}(\zeta)} \frac{\partial}{\partial \zeta}\left(1-\zeta^{2}\right) \frac{\partial}{\partial \zeta}
$$

with $\mathcal{L}_{0}=(D-3) / 2$, while the weight function $W_{0}$ by

$$
W_{0}(\zeta)=(1-\zeta)^{(D-5) / 2}(1+\zeta)^{1 / 2}
$$

The $V^{(i)} \equiv V^{(i)}\left(r_{0} \sqrt{(1+\zeta) / 2}\right), i=1+, 3+$, are the even singlet and triplet nucleonnucleon potentials. The kernels $f_{(0)}^{n}\left(\zeta, \zeta^{\prime}\right), n=S, S^{\prime}$ result from the projection on to the $r_{i j}$ space. More details concerning these kernels and other technical points can be found in Refs. 16, 17.

Once the components $P_{i j}^{n}\left(\zeta, r_{0}\right)$ are found, one can construct the various symmetries for the bound state wave function $\Psi_{3}([18])$. However, in the present calculation we used only the space symmetric part i.e.,

$$
\left\langle\boldsymbol{\rho}, \mathbf{r} \mid \Psi_{3}\right\rangle=\frac{\psi_{3}(\boldsymbol{\rho}, \mathbf{r})}{\sqrt{2}}\left[\left|\left(\left(s_{1} s_{2}\right) 1 s_{3}\right) \frac{1}{2} \sigma ;\left(\left(t_{1} t_{2}\right) 0 t_{3}\right) \frac{1}{2} \tau\right\rangle-\left|\left(\left(s_{1} s_{2}\right) 0 s_{3}\right) \frac{1}{2} \sigma ;\left(\left(t_{1} t_{2}\right) 1 t_{3}\right) \frac{1}{2} \tau\right\rangle\right] .
$$

It is emphasised here that the IDEA for the three-body case and for S-projected potentials, is equivalent to the exact Faddeev equations.

\section{E. Electron scattering}

The scattering of the electron on the $i$-th nucleon, is described by the $t$-matrix

$$
t_{i}(z)=V_{i}+V_{i} G_{0}(z) t_{i}(z)
$$

where

$$
V_{i}=-\hat{Z}_{i} e^{2} / r_{i}
$$

and $G_{0}(z)$ is the free Green function. Using (31) we can rewrite Eq. (8) as a sum of Faddeev components, viz.,

$$
T=\sum_{i=1}^{3} T_{i}
$$

with

$$
T_{i}=t_{i}+t_{i} G_{0} \sum_{j \neq i} T_{j}
$$


Iterative solution of these equations provides the multiple scattering series

$$
T=t_{1}+t_{2}+t_{3}+t_{1} G_{0} t_{2}+t_{2} G_{0} t_{1}+t_{1} G_{0} t_{3}+t_{3} G_{0} t_{1}+t_{2} G_{0} t_{3}+t_{3} G_{0} t_{2}+\cdots .
$$

Since the average energy of the Coulomb interaction of the electron with the nucleons is of atomic order of magnitude $(\sim 10 \mathrm{eV})$ and the average collision energy is $\langle\kappa \Theta\rangle \sim 10^{3} \mathrm{eV}$, we can omit the higher order rescattering terms in Eq. (34), that is, we use the following approximation [19]

$$
T \approx t_{1}+t_{2}+t_{3} .
$$

For the same reason we can apply the Born approximation for the Coulomb $t$-matrix, i.e.

$$
t_{c}\left(\mathbf{p}^{\prime}, \mathbf{p} ; Q\right) \approx-\frac{Q e^{2}}{2 \pi^{2}\left(\mathbf{p}-\mathbf{p}^{\prime}\right)^{2}},
$$

where $t_{c}$ is the two-body Coulomb $t$-matrix for an electron scattered off the charge $Q e$.

To obtain matrix elements of the $T$-operator (35), we use the following basis states

$$
|\boldsymbol{\rho}, \mathbf{r}, \mathbf{p} ; \chi \eta\rangle \equiv\left|\boldsymbol{\rho}, \mathbf{r}, \mathbf{p} ;\left(\left(s_{1} s_{2}\right) s_{12} s_{3}\right) \frac{1}{2} \sigma ;\left(\left(t_{1} t_{2}\right) t_{12} t_{3}\right) \frac{1}{2} \frac{1}{2}\right\rangle,
$$

where $\mathbf{p}$ is the electron momentum with respect to the center of mass of the nucleons. Using the space displacement operators [9] $\exp \left(-i \boldsymbol{\Delta}_{i} \mathbf{p}\right)$, where $\boldsymbol{\Delta}_{i}$ is a vector directed from the center of mass to the $i$-th nucleon, we also construct the shifted basis states,

$$
\left|\boldsymbol{\rho}, \mathbf{r}, \mathbf{p}_{i} ; \chi \eta\right\rangle=\exp \left(-i \boldsymbol{\Delta}_{i} \mathbf{p}\right)|\boldsymbol{\rho}, \mathbf{r}, \mathbf{p} ; \chi \eta\rangle
$$

which differ from (36) in the sense that the electron has the same momentum $\mathbf{p}_{i}=\mathbf{p}$ but with respect to the $i$-th nucleon (not to the center of mass). Thus, we obtain

$$
\left\langle\boldsymbol{\rho}^{\prime}, \mathbf{r}^{\prime}, \mathbf{p}^{\prime} ; \chi^{\prime} \eta^{\prime}|T(z)| \boldsymbol{\rho}, \mathbf{r}, \mathbf{p} ; \chi \eta\right\rangle=\delta_{\chi^{\prime} \chi} \delta \eta^{\prime} \eta \delta\left(\boldsymbol{\rho}^{\prime}-\boldsymbol{\rho}\right) \delta\left(\mathbf{r}^{\prime}-\mathbf{r}\right) T_{\mathbf{p}^{\prime} \mathbf{p}}^{\eta}(\boldsymbol{\rho}, \mathbf{r} ; z)
$$

with

$$
T_{\mathbf{p}^{\prime} \mathbf{p}}^{\eta}(\boldsymbol{\rho}, \mathbf{r} ; z)=\sum_{m=1}^{3} \exp \left[i\left(\mathbf{p}-\mathbf{p}^{\prime}\right) \boldsymbol{\Delta}_{m}\right] t_{c}\left(\mathbf{p}^{\prime}, \mathbf{p} ; Q_{m}^{\eta}\right),
$$

where the effective charges are

$$
Q_{m}^{\eta}=\left\langle\eta\left|\hat{Z}_{m}\right| \eta\right\rangle
$$

Using the $6 j$-symbols [20] for recoupling the isospins, we obtain

$$
\begin{array}{rlrl}
Q_{1}^{0} & =\frac{1}{2}, & Q_{2}^{0}=\frac{1}{2}, & Q_{3}^{0}=1, \\
Q_{1}^{1}=\frac{5}{6}, & Q_{2}^{1}=\frac{5}{6}, & Q_{3}^{1}=\frac{1}{3},
\end{array}
$$

where the upper index 0 designates the $\left|\left(\left(t_{1} t_{2}\right) 0 t_{3}\right) \frac{1}{2} \frac{1}{2}\right\rangle$ and 1 the $\left|\left(\left(t_{1} t_{2}\right) 1 t_{3}\right) \frac{1}{2} \frac{1}{2}\right\rangle$ states. 


\section{RESULTS AND CONCLUSIONS}

The effective $p d$ potential obtained using the Marchenko inverse scattering theory, described in Sec. IIC, for the $\ell=0$ partial wave, is plotted in Fig. II. This potential reproduces the experimental $n d$ phase shifts as the Coulomb $p d$ interaction has been treated separately as described in Eq. (15). Since these phase shifts, however, are available only at low energies we use for large values of $k$ the phase shifts obtained via the Faddeev equations [21]. The oscillations in the interaction region are due to the opening of the break-up channel as well as to the behaviour of the phase shifts at large $k$ values $(k \geq 1000 \mathrm{MeV})$.

The wave function of the ${ }^{3} \mathrm{He}-$ nucleus was obtained by solving the system (27) with the Malfliet-Tjon I-III (MT I-III) nucleon-nucleon potential [10] as input. The binding energy obtained is $8.86 \mathrm{MeV}$ while the root mean square radius is $1.685 \mathrm{fm}$. However, for our final calculations we used the energy release in the reaction (2), viz.,

$$
\mathcal{E}=E_{3}-E_{2},
$$

correponding to the difference between the experimental binding energies of the deuteron and ${ }^{3} \mathrm{He}$, namely $E_{2}=2.224574 \mathrm{MeV}$ and $E_{3}=7.718109 \mathrm{MeV}$ [22].

The rate of the reaction (2) can be presented in the following factorized form (see the Appendix)

$$
\langle\mathcal{R}\rangle=n_{p} n_{d} n_{e}\langle\Sigma\rangle,
$$

where the quantity $\langle\Sigma\rangle$ is analogous to $\langle\sigma v\rangle$ generally used in two-body reaction theories [7]. The calculated rates for different temperatures of the plasma are presented in Table I, where the results are given in units of $\mathrm{cm}^{6} \mathrm{~mole}^{-2} \mathrm{sec}^{-1}$ [23]. These units are obtained when instead of $n_{p} n_{d} n_{e}$ we multiply $\langle\Sigma\rangle$ by $N_{A}^{2}$, i.e. the Avogadro number squared. The second column of the Table I contains the values of

$$
\mathcal{R}_{e}=N_{A}^{2}\langle\Sigma\rangle
$$

while the third contains the rate for the radiative capture (11) which is also presented in the form

$$
\mathcal{R}_{\gamma}=N_{A}\langle\sigma v\rangle,
$$

instead of $n_{p} n_{d}\langle\sigma v\rangle$, that is in $\mathrm{cm}^{3} \mathrm{~mole}^{-1} \mathrm{sec}^{-1}$ units (the data are taken from Ref. [23]).

In order to assess the importance of the nonradiative process (2) as compared to the radiative capture (1) when ${ }^{3} \mathrm{He}$ nuclei are generated, we must compare the rates for these two processes per $\mathrm{cm}^{3}$ per sec. This requires the knowlege of the particle densities $n_{p}, n_{d}$, and $n_{e}$, and therefore we need to specify the plasma conditions. However, by considering the ratio of the two rates, viz.,

$$
\text { ratio }=n_{e} \frac{\langle\Sigma\rangle}{\langle\sigma v\rangle}
$$

only one unknown parameter remains, namely the electron density $n_{e}$. We calculated this ratio for the reaction rates for (2) and (1) by using the value $n_{e}=100 N_{A} \mathrm{~cm}^{-3}$ corresponding 
to the solar interior plasma [1]. The calculated ratio is given in the fourth column of Table I. The ratio for any other electron density $n_{e}$ can be obtained by simply multiplying these values by $n_{e} / 100 N_{A}$.

It is seen that in the solar $p p$-chain the nonradiative fusion (2) plays, apparently, a minor

role. However, at the early stages of the universe when $n_{e} / N_{A} \gg 100$, this reaction must have been significant.

\section{Acknowledgements}

One of us (S.A.R) gratefully acknowledges financial support from the University of South Africa and the Joint Institute for Nuclear Research, Dubna. 


\section{APPENDIX A: REACTION RATE: EXPLICIT FORMULA}

Due to the very low nuclear energies involved we shall use only $S$-wave components of the wave functions describing the deuteron, $\psi_{2}$, the $p d$ relative motion, $\varphi_{\mathbf{k}}$, and the nucleus ${ }^{3} \mathrm{He}, \psi_{3}$. These functions depend only on the moduli of the corresponding vector variables. However, due to the antisymmetrization, the dependence on the angle between the vectors $\boldsymbol{\rho}$ and $\mathbf{r}$ is also present.

The fixed scatterer $T$-matrix (8) depends on all four angles that define the directions of the vectors $\boldsymbol{\rho}$ and $\mathbf{r}$ which are involved in (37) in the form of the following combinations

$$
\begin{aligned}
& \boldsymbol{\Delta}_{1}=\frac{1}{2} \mathbf{r}+\frac{1}{3} \boldsymbol{\rho}, \\
& \boldsymbol{\Delta}_{2}=\frac{1}{3} \boldsymbol{\rho}-\frac{1}{2} \mathbf{r}, \\
& \boldsymbol{\Delta}_{3}=-\frac{2}{3} \boldsymbol{\rho} .
\end{aligned}
$$

Thus, the transition matrix (9) is a a 6-dimensional integral. The final averaging procedure involves 9 more integrations,

$$
\langle\mathcal{R}\rangle=n_{p} n_{d} n_{e}(2 \pi)^{7} \iiint d \mathbf{k} d \mathbf{p} d \mathbf{p}^{\prime} \delta\left(E_{f}-E_{i}\right)\left|\left\langle\Psi_{3}, \mathbf{p}^{\prime}|T| \Psi_{\mathbf{k}}, \mathbf{p}\right\rangle\right|^{2} N_{\mathbf{k}} N_{\mathbf{p}} .
$$

The transition matrix $\left\langle\Psi_{3}, \mathbf{p}^{\prime}|T| \Psi_{\mathbf{k}}, \mathbf{p}\right\rangle$ depends on $\left(\mathbf{p}^{\prime}-\mathbf{p}\right)$ and $|\mathbf{k}|$. Moreover the Maxwell distributions are isotropic. Hence, the 5-dimensional integration over $d \Omega_{\mathbf{k}} d \Omega_{\mathbf{p}^{\prime}} d \phi_{\mathbf{p}}$ is trivial and results in the factor $32 \pi^{3}$. One more integration, over the final momentum, is performed with the help of the energy conserving $\delta$-function and the 9-dimensional integral is reduced to a 3-dimensional one. Thus, finally we obtain

$$
\langle\mathcal{R}\rangle=n_{p} n_{d} n_{e}\langle\Sigma\rangle
$$

where $\langle\Sigma\rangle$ is defined by

$$
\begin{aligned}
\langle\Sigma\rangle & =\frac{4 \pi^{3} e^{4} c}{3 \sqrt{m \mu^{3}} \kappa^{3} \Theta^{3}} \int_{0}^{\infty} \int_{0}^{\infty} d k d p k^{2} p^{2} \exp \left(-\frac{k^{2}}{2 \mu \kappa \Theta}-\frac{p^{2}}{2 m \kappa \Theta}\right) \\
& \times \int_{-1}^{+1} d x \frac{Q}{q^{4}} \mid \int_{0}^{\infty} \int_{0}^{\infty} d r d \rho \int_{0}^{2 \pi} \int_{0}^{2 \pi} d \phi_{r} d \phi_{\rho} \int_{-1}^{+1} \int_{-1}^{+1} d y d z r^{2} \rho^{2} \psi_{3}(\rho, r) \\
& \times\left\{\left[\varphi_{k}\left(\sqrt{9 r^{2} / 16+\rho^{2} / 4-3 \mathbf{r} \cdot \boldsymbol{\rho} / 4}\right) \psi_{2}\left(\sqrt{r^{2} / 4+\rho^{2}+\mathbf{r} \cdot \boldsymbol{\rho}}\right)\right.\right. \\
& \left.+\varphi_{k}\left(\sqrt{9 r^{2} / 16+\rho^{2} / 4+3 \mathbf{r} \cdot \boldsymbol{\rho} / 4}\right) \psi_{2}\left(\sqrt{r^{2} / 4+\rho^{2}-\mathbf{r} \cdot \boldsymbol{\rho}}\right)\right] \\
& \times\left[3 \exp \left(\frac{i q \rho y}{3}+\frac{i q r z}{2}\right)+3 \exp \left(\frac{i q \rho y}{3}-\frac{i q r z}{2}\right)+2 \exp \left(\frac{-i 2 q \rho y}{3}\right)\right] \\
& \left.+\varphi_{k}(\rho) \psi_{2}(\rho)\left[2 \exp \left(\frac{i q \rho y}{3}+\frac{i q r z}{2}\right)+2 \exp \left(\frac{i q \rho y}{3}-\frac{i q r z}{2}\right)+4 \exp \left(\frac{-i 2 q \rho y}{3}\right)\right]\right\}\left.\right|^{2},
\end{aligned}
$$

where the scalar product $\mathbf{r} \cdot \boldsymbol{\rho}$ is

$$
\mathbf{r} \cdot \boldsymbol{\rho}=r \rho\left[\sqrt{\left(1-y^{2}\right)\left(1-z^{2}\right)} \cos \left(\phi_{r}-\phi_{\rho}\right)+y z\right],
$$


while

$$
Q=\sqrt{2 m \mathcal{E}+\frac{m}{\mu} k^{2}+p^{2}},
$$

and

$$
q=\sqrt{Q^{2}+p^{2}-2 Q p x} .
$$




\section{REFERENCES}

[1] J.N. Bachall, Neutrino Astrophysics (Cambridge University Press, New York, 1989).

[2] Primordial Nucleosynthesis, Proceedings of the international workshop, October 6 - 8, 1989, Chapel Hill, USA, ed.W.J.Thompson (World Scientific, Singapore, 1990).

[3] R.A. Malaney, Nuclei in the Cosmos, ed.H.Oberhummer, (Springer-Verlag, Berlin, 1991), p. 127.

[4] M.S. Turner, Particle Physics, Astrophysics $\&$ Cosmology, Proc. of the XXII SLAC Summer Institute on Particle Physics, August 8-19, 1994, Stanford, USA; p. 1.

[5] E.W. Kolb et al., Phys. Rev. Lett. 67 (1991) 533.

[6] S. Dodelson, G. Gyuk, and M. S. Turner, Phys. Rev. D 49 (1994) 5068.

[7] Nuclei in the Cosmos, ed.H.Oberhummer, (Springer-Verlag, New-York, 1991).

[8] H. Leeb and H. Oberhummer, Astrophysics and Space Science 320 (1995) 117.

[9] M. L. Goldberger and K. M. Watson, Collision Theory, (John Wiley \& Sons, New York, 1964).

[10] R. A. Malfliet and J. A. Tjon, Nucl. Phys. A127 (1969) 161; Ann. Phys. (N.Y.) 61 (1970) 425.

[11] S. A. Rakityansky, S. A. Sofianos, and K. Amos, Nuovo Cim. B 111 (1996) 363.

[12] E. E. Salpeter, Australian J. of Phys. 7 (1954) 373.

[13] Z.S. Agranovich and V. A. Marchenko, The inverse problem of the scattering theory (Gordon \& Breach, New York, 1963).

[14] K. Chadan and P. C. Sabatier, Inverse problems in quantum scattering theory (SpringerVerlag, New York, 1977).

[15] M. Fabre de la Ripelle, in Models and Methods in Few-Body Physics, Vol. 273 of Lecture Notes in Physics, ed. L.S. Fereira, A. C. Fonseca, and L. Streit (Springer-Verlag, New York, 1987), pp 283-323.

[16] M. Fabre de la Ripelle, H. Fiedeldey, and S. A. Sofianos, Phys. Rev. C 38 (1988), 449.

[17] W. Oehm, S. A. Sofianos, H. Fiedeldey, and M. Fabre de la Ripelle, Phys. Rev. C 44 (1991) 81.

[18] W. Oehm, S. A. Sofianos, H. Fiedeldey, and M. Fabre de la Ripelle, Phys. Rev. C 43 (1991) 25.

[19] J. R. Taylor, Scattering Theory ( John Wiley \& Sons, Inc., New York, 1972).

[20] D. A. Varshalovich, A. N. Moskalev, V. K. Khersonskii, Quantum Theory of Angular Momentum (World Scientific, Singapore, 1988).

[21] E. O. Alt, L. L. Howell, M. Rauh, and S. A. Sofianos, Phys. Rev. C 49 (1994) 176.

[22] A. H. Wapstra, G. Andi, Nucl Phys. A432 (1985) 1.

[23] G. R. Caughlan, W. A. Fowler, Atomic Data and Nuclear Data Tables, Vol. 40 (1988), pp. 283-334. 


\section{FIGURES}

FIG. 1. The Jacobi coordinates for the $e+p+d$ system.

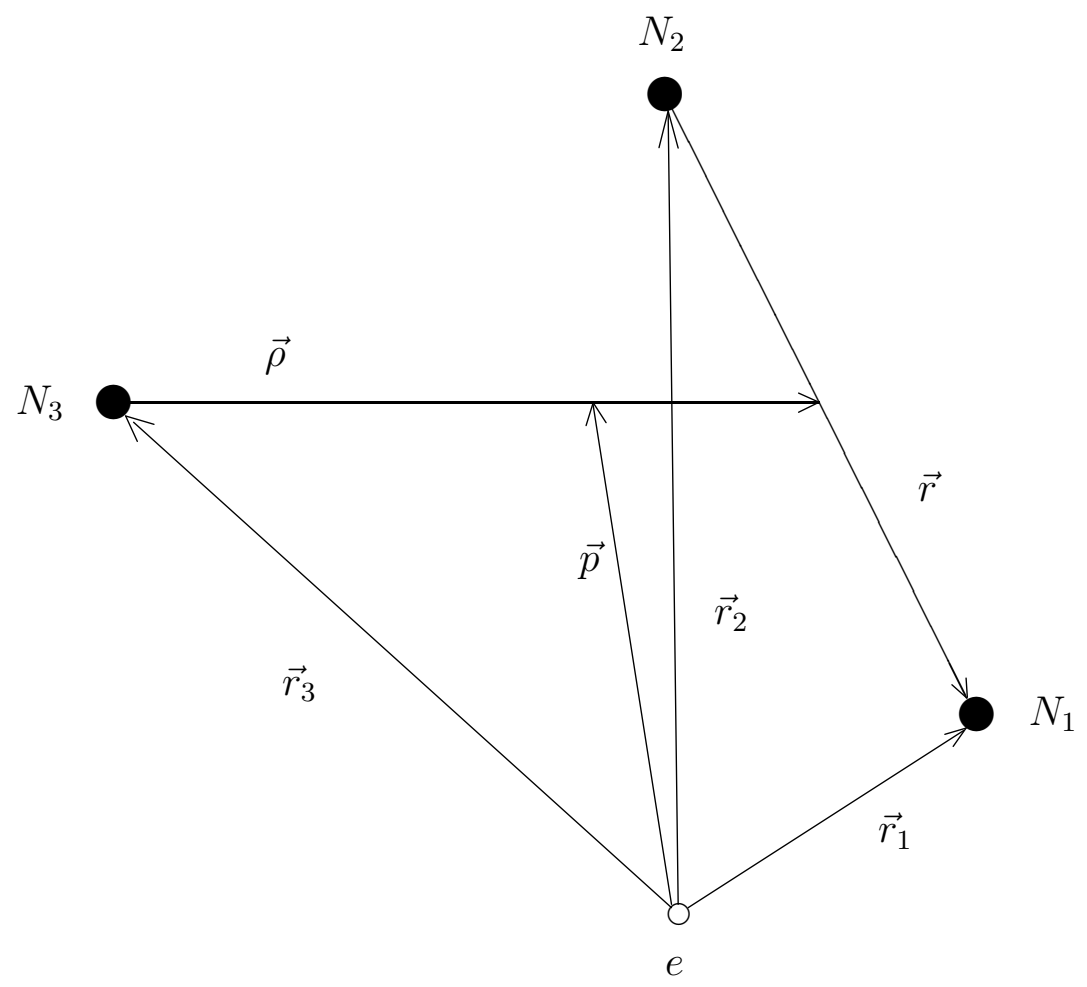


FIG. 2. The $n d$ effective interaction obtained using the Marchenko inverse scattering method.

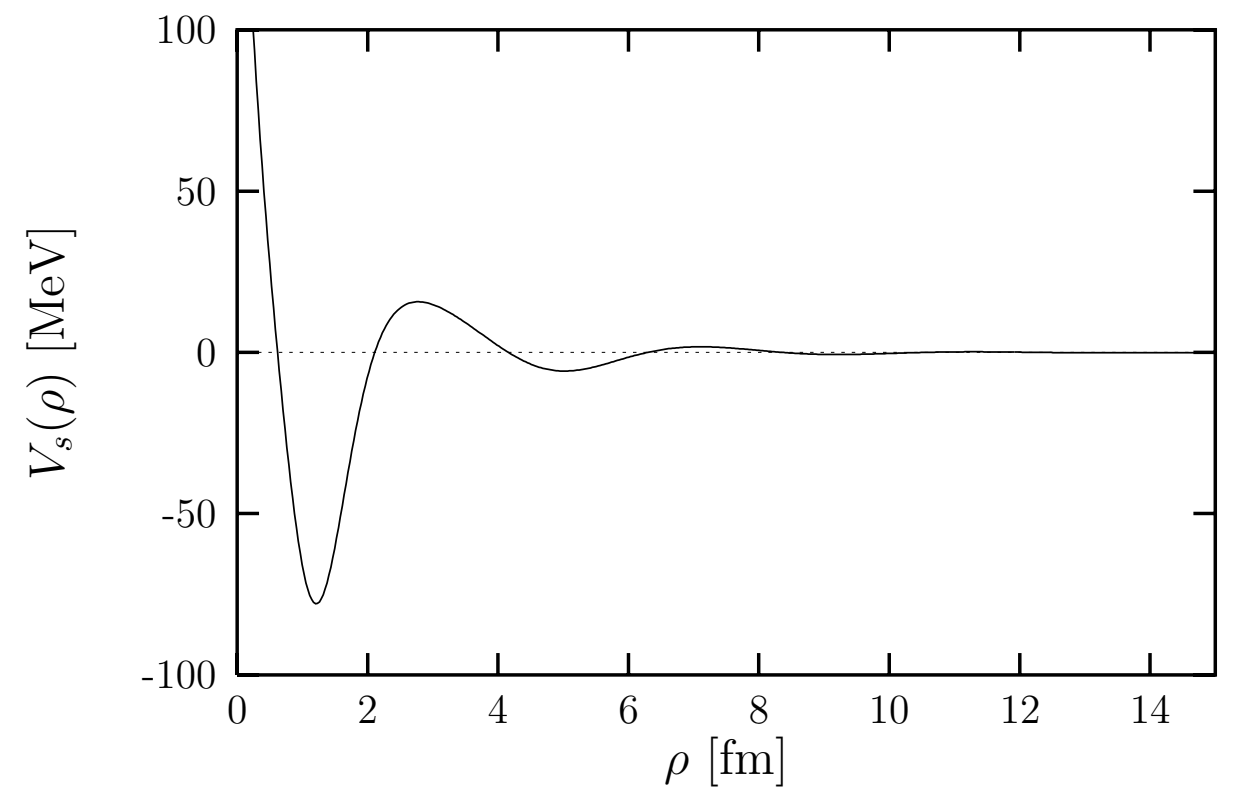




\section{TABLES}

TABLE I. Nonradiative, $\mathcal{R}_{e}=\left\langle\mathcal{R}\left(p d e \rightarrow e^{3} \mathrm{He}\right)\right\rangle$, and radiative, $\mathcal{R}_{\gamma}=\left\langle\mathcal{R}\left(p d \rightarrow{ }^{3} \mathrm{He} \gamma\right)\right\rangle$, capture rates as functions of the plasma temperature $T_{6}$. The fourth column shows their ratio for $n_{e}=100 N_{A} \mathrm{~cm}^{-3}$. The temperature $T_{6}$ is in $10^{6}{ }^{\circ} \mathrm{K}$ units, and the reaction rates in $\mathrm{cm}^{6}$ mole $^{-2} \mathrm{sec}^{-1}$ and $\mathrm{cm}^{3}$ mole ${ }^{-1} \mathrm{sec}^{-1}$ respectively. The data for $\left\langle\mathcal{R}\left(p d \rightarrow{ }^{3} \mathrm{He} \gamma\right)\right\rangle$ are taken from Ref. [23].

\begin{tabular}{|c|c|c|c||c|c|c|c|}
\hline \hline \hline$T_{6}$ & $\mathcal{R}_{e}$ & $\mathcal{R}_{\gamma}$ & Ratio & $T_{6}$ & $\mathcal{R}_{e}$ & $\mathcal{R}_{\gamma}$ & Ratio \\
\hline 1 & $0.357(-16)$ & $0.164(-10)$ & $0.218(-3)$ & 15 & $0.153(-7)$ & $0.132(-1)$ & $0.116(-3)$ \\
2 & $0.423(-13)$ & $0.228(-7)$ & $0.186(-3)$ & 16 & $0.201(-7)$ & $0.176(-1)$ & $0.114(-3)$ \\
3 & $0.124(-11)$ & $0.741(-6)$ & $0.167(-3)$ & 18 & $0.324(-7)$ & $0.292(-1)$ & $0.111(-3)$ \\
4 & $0.103(-10)$ & $0.658(-5)$ & $0.157(-3)$ & 20 & $0.486(-7)$ & $0.453(-1)$ & $0.107(-3)$ \\
5 & $0.459(-10)$ & $0.309(-4)$ & $0.147(-3)$ & 25 & $0.108(-6)$ & 0.109 & $0.990(-4)$ \\
6 & $0.144(-9)$ & $0.100(-3)$ & $0.144(-3)$ & 30 & $0.198(-6)$ & 0.211 & $0.938(-4)$ \\
7 & $0.354(-9)$ & $0.255(-3)$ & $0.139(-3)$ & 40 & $0.474(-6)$ & 0.557 & $0.851(-4)$ \\
8 & $0.746(-9)$ & $0.552(-3)$ & $0.135(-3)$ & 50 & $0.863(-6)$ & $0.111(+1)$ & $0.777(-4)$ \\
9 & $0.139(-8)$ & $0.106(-2)$ & $0.131(-3)$ & 60 & $0.135(-5)$ & $0.188(+1)$ & $0.718(-4)$ \\
10 & $0.238(-8)$ & $0.185(-2)$ & $0.129(-3)$ & 70 & $0.191(-5)$ & $0.286(+1)$ & $0.668(-4)$ \\
11 & $0.381(-8)$ & $0.301(-2)$ & $0.127(-3)$ & 80 & $0.253(-5)$ & $0.406(+1)$ & $0.623(-4)$ \\
12 & $0.573(-8)$ & $0.463(-2)$ & $0.124(-3)$ & 90 & $0.318(-5)$ & $0.545(+1)$ & $0.583(-4)$ \\
13 & $0.824(-8)$ & $0.680(-2)$ & $0.121(-3)$ & 100 & $0.387(-5)$ & $0.703(+1)$ & $0.550(-4)$ \\
14 & $0.114(-7)$ & $0.963(-2)$ & $0.118(-3)$ & 200 & $0.106(-4)$ & $0.311(+2)$ & $0.151(-3)$ \\
\hline \hline
\end{tabular}

